\title{
Paroxysmal nocturnal hemoglobinuria: pathophysiology, natural history and treatment options in the era of biological agents
}

\author{
Antonio M Risitano \\ Bruno Rotoli \\ Hematology, Department of \\ Biochemistry and Medical \\ Biotechnologies, Federico II \\ University of Naples, Italy
}

Correspondence: Antonio M Risitano Hematology, DBBM, Federico II University of Naples, Via Pansini 5-80I3I Naples, Italy

Tel +3908I 7462037

Fax +3908I 7462165

Email amrisita@unina.it

\begin{abstract}
Paroxysmal nocturnal hemoglobinuria (PNH) is a clonal non-malignant hematological disease characterized by the expansion of hematopoietic stem cells (HSCs) and progeny mature cells, whose surfaces lack all the proteins linked through the glycosyl-phosphatidyl inositol anchor. This defect arises from an acquired somatic mutation in the X-linked phosphatidylinositol glycan class $A$ gene, with subsequent clonal expansion of the mutated HSCs as a result of a concomitant, likely immune-mediated, selective pressure. The disease is characterized by complement-mediated chronic intravascular hemolysis, resulting in hemolytic anemia and hemosiderinuria; capricious exacerbations lead to recurrent gross hemoglobinuria. Additional cardinal manifestations of PNH are a variable degree of bone marrow failure and an intrinsic propensity to thromboembolic events. The disease is markedly invalidating, with chronic symptoms requiring supportive therapy - usually including periodical transfusions; possible life-threatening complications may also ensue. The biology of PNH has been progressively elucidated in the past few years, but therapeutic strategies remained unsatisfactory for decades, the only exception being stem cell transplantation, which is restricted to selected patients and retains significant morbidity and mortality. Recently, a biological agent to treat PNH has been developed - the terminal complement inhibitor eculizumab - which has been tested in a number of clinical trials, with exciting results. All the data from worldwide clinical trials confirm that eculizumab radically modifies the symptoms, the biology, and the natural history of $\mathrm{PNH}$, strongly improving the quality of life of PNH patients.
\end{abstract}

Keywords: paroxysmal nocturnal hemoglobinuria, GPI-AP, PIG-A, complement, eculizumab

\section{Introduction}

Paroxysmal nocturnal hemoglobinuria (PNH), first described in the 18th century, is characterized by the typical clinical triad of hemolytic anemia, bone marrow failure, and propensity to thromboembolism (Rotoli and Luzzatto 1989; Dunn et al 2000; Rotoli et al 2006; Hill et al 2007a). It is a rare disease, with a worldwide prevalence estimated in the range of 1-5 cases per million (Rosse 1996) regardless of ethnicity; an increased prevalence is reported in some regions which also harbor higher incidence of aplastic anemia (eg, Thailand and some other Asian countries) (Pramoonjago et al 1999). While the original denomination focused on a single component of the diseasenamely intravascular hemolysis and subsequent hemoglobinuria - its complexity emerged in subsequent studies, and remained a riddle for generations of investigators. In the past 3 decades most pathogenic mechanisms have been unraveled, although some biological aspects have not been elucidated yet.

$\mathrm{PNH}$ is a hemolytic anemia resulting from the clonal expansion of one or a few abnormal hematopoietic stem cells (HSCs) (Oni et al 1970). This defect was identified by genetic studies in the $1990 \mathrm{~s}$ as a mutation in the X-linked phosphatidyl-inositol 
glycan class A (PIG-A) gene (Takeda et al 1993; Luzzatto et al 1997). PNH is therefore an acquired genetic blood disorder; however, the $P I G-A$ mutation is likely insufficient to cause the disease and additional events may be involved (Rotoli and Luzzatto 1989), as noted below. Indeed, the genetic abnormality does not explain the entire clinical phenotype of $\mathrm{PNH}$, especially marrow failure and thrombosis. Here we will review the natural history of $\mathrm{PNH}$, focusing on clinical manifestations and their specific pathogenic mechanisms, aiming to give a theoretical background for targeted therapeutic strategies. In addition, we will discuss the clinical results from recent international trials using the complement inhibitor eculizumab $\left(\right.$ Soliris $^{\circledR}$ ) as treatment for PNH patients.

\section{Pathophysiology}

As mentioned above, PNH has a well-recognized genetic cause that is neither inherited nor transmitted to the progeny (Takeda et al 1993; Luzzatto et al 1997). It develops through a somatic mutation in the $P I G-A$ gene occurring in HSC(s), which originate progeny mature blood cells with the bizarre feature of a lack of several proteins from their surface. This abnormality was first described in the 1980s (Nicholson-Weller et al 1983; Selvaraj et al 1988), and rapidly became the hallmark of $\mathrm{PNH}$, although its relationship with the pathophysiology of the disease remained obscure at that time. Subsequently, it became clear that all the proteins missing from the PNH cell surface share a common mechanism for attaching to the cell membrane, which was identified in a specific glycolipid structure named glycosylphosphatidyl inositol (GPI) anchor (Mahoney et al 1992). In 1993, using complementation of GPI-anchored protein deficient cell lines and expression cloning, Kinoshita and colleagues first isolated the cDNA of the $P I G-A$ gene (Takeda et al 1993; Miyata et al 1993). $P I G-A$ is a housekeeping gene located on the short arm of the X chromosome (Xp22.1); the organization of the genomic gene was described in 1994 (Bessler et al 1994). The PIG- $A$ gene encodes an enzyme essential, in combination with at least two other proteins, to transfer $\mathrm{N}$-acetyl glucosamine to phosphatidyl inositol; this is the very first step of the glycosyl-phosphatidyl inositol (GPI)-anchor biosynthesis (Hirose et al 1992; Takahashi et al 1993). All mutations identified in PNH patients affect the $P I G$ - $A$ gene; no abnormalities were found in any of the other numerous genes involved in the subsequent metabolic steps of the GPI-anchor synthesis. In fact, $P I G-A$ is the only $\mathrm{X}$-linked gene, and a single mutational event is sufficient to impair GPI-anchor biosynthesis (also in females, as a result of $\mathrm{X}$-chromosome functional inactivation). As an exception, an autosomal recessive inherited GPI deficiency resulting from a mutation of the $P I G-M$ gene has been recently described in two kindreds; however, the phenotype was clearly different from that of $\mathrm{PNH}$, with a partial GPI deficiency resulting in a clinical syndrome characterized by propensity to thrombosis and seizures, in the absence of significant hemolysis (Almeida et al 2006; Almeida et al 2007).

The role of the GPI-anchor in cell biology seems to be very important, as supported by its high conservation in eukaryotic cells, including yeast and trypanosome; however, the functional implications of the GPI-anchoring of proteins, and of the GPI-anchor itself, are not completely understood. It is known that GPI-anchored proteins (GPI-APs) are not randomly distributed on the cell surface, but rather constitute membrane subdomains enriched in specific lipids, such as cholesterol and sphyngolipids; these structures, called rafts, seem to be involved in membrane trafficking and remodeling, including capping and endo-, exo-, and potocytosis, as well as in signal transduction (eg, immunological synapse) (Goebel et al 2002).

As a result of the GPI-anchor deficiency, PNH cells lack all GPI-APs on their surface (Figure 1A); so far, more than 25 different proteins sharing the GPI-anchoring system have been described on blood cells (Table 1) (Rotoli et al 1993), their specific functions being only partially known. However, some GPI-APs are functionally well-described proteins, and have been essential to fully unravel the pathogenic mechanisms of some clinical manifestations of PNH (Figure 2). In fact, among the GPIAPs there are two complement-regulatory proteins, namely CD59/MIRL (Membrane Inhibitor of Reactive Lysis) (Zalman et al 1987; Okada et al 1990) and CD55/DAF (Decay Accelerating Factor) (Nicholson-Weller et al 1983; Nicholson-Weller et al 1985). They act at different levels of the complement cascade (Figure 3): CD55 controls early complement activation, inhibiting $\mathrm{C} 3$ and $\mathrm{C} 5$ convertases (Burge et al 1981; Medof et al 1984), while CD59 interferes with the terminal effector complement, blocking the incorporation of $\mathrm{C} 9$ onto the $\mathrm{C} 5 \mathrm{~b}-\mathrm{C} 8$ complex, forming the membrane attack complex (MAC) (Shin et al 1986; Zalman et al 1986). The deficiency of such proteins leads to the increased susceptibility of PNH red blood cells (RBCs) to complement mediated lysis, resulting in the typical intravascular hemolysis (Holguin et al 1989). In addition, hypersensitivity to complement activation in vitro leads to red cell destruction upon serum acidification, a feature 


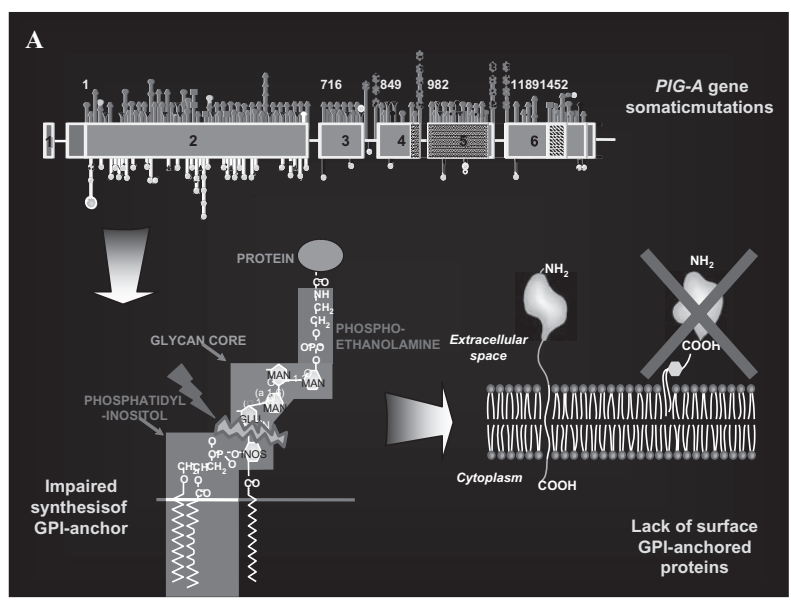

B

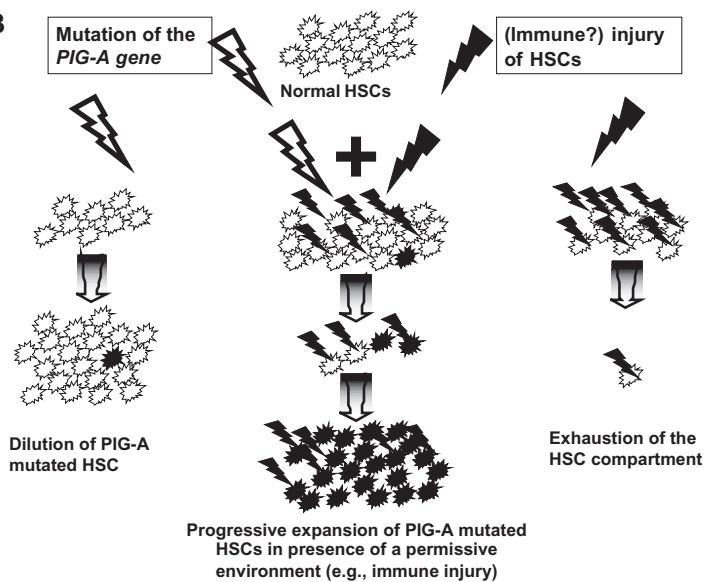

Figure I Paroxysmal nocturnal hemoglobinuria (PNH) pathophysiology. A. PIG-A mutations: mutation occurring in the PIG-A gene may affect the synthesis or the function of the encoded protein, resulting in the impairment of the first step of GPI-anchor biosynthesis. As a result, all proteins linked to the cellular membrane through this mechanism are lacking from the surface. B. The dual pathophysiology theory: PIG-A gene mutation may occur without provoking the $\mathrm{PNH}$ disease. To induce the clonal expansion necessary for developing clinical $\mathrm{PNH}$, additional factors should occur, which create conditions permissive to expansion of mutated cells. Such extrinsic factors are postulated similar to those inducing stem cell exhaustion in aplastic anemia, which include immune mediated attack of hematopoiesis.

utilized as a diagnostic assay for PNH for several decades (the HAM test, recently replaced by flow cytometry). A low level of complement activation is a constant physiological phenomenon greatly amplified during inflammatory or infectious diseases; thus, PNH erythrocytes chronically undergo intravascular hemolysis in PNH patients due to CD55 and CD59 deficiency, with a lifespan reduced to $10 \%$ compared with that of normal RBCs (Wiedmer et al 1993). GPI-AP deficiency on RBCs may be complete (PNH type III cells) or partial (PNH type II cells), resulting in different sensitivity to complement mediated lysis, as initially described by Rosse (Rosse and Dacie 1966; Rosse 1990). However, particular aspects of hemolysis in $\mathrm{PNH}$ are still debated; for instance, it is still unclear why exacerbations occur mainly during the night. In a functional hierarchy, CD59 was demonstrated as playing a major role (Wilcox et al 1991). This is also supported by the observation of rare patients harboring inherited deficiency of a single molecule (Yamashina et al 1990; Shichishima et al 1999); in fact, a unique CD59-deficient patient had a PNH-like picture, whereas subjects deficient in CD55 on red cell membrane (ie, those carrying the rare Inab blood group phenotype) showed no clinically significant hemolysis.

CD55 and CD59 are deficient on RBCs, but also on white blood cells (WBCs) and platelets (Nicholson-Weller et al 1985); however, as far as WBCs are concerned, there is no evidence of reduced leukocyte lifespan in $\mathrm{PNH}$ patients, probably because nucleated cells have an additional transmembrane (ie, non GPI-linked) complement inhibitor (CD46) (Christmas et al 2006). In contrast, uncontrolled complement activation on platelets has been hypothesized as a possible explanation for another clinical hallmark of $\mathrm{PNH}$, namely the propensity to thromboembolic events (Figure 2). In fact, complement activation might lead to platelet activation and aggregation, enhancing clot formation; however, no demonstration of such a mechanism has been provided up to now, and other different mechanisms have been postulated. In $\mathrm{PNH}$, microvesicles are known to be released upon hemolysis and complement activation from RBCs (Hugel et al 1999), WBCs (monocytes) and platelets (Wiedmer et al 1993; Simak et al 2004), and even from the endothelium; their procoagulant action is commonly accepted, but their specific role in the pathophysiology of thromboembolisms in PNH still needs to be documented. An additional mechanism of thrombophilia in PNH might be the impairment of the fibrinolytic system, due to the lack of membrane-bound urokinase-type plasminogen activator receptor (uPAR), which is GPI-linked, and to the excess of its soluble form (Ninomiya et al 1997; Sloand et al 2006). Finally, thrombophilia in PNH may arise from the build-up of cell-free plasma hemoglobin due to hemolysis (Olsen et al 1996; Schafer et al 2004). This may occur through ability of free hemoglobin to scavenge nitric oxide from plasma, blocking its inhibitory action on platelet aggregation and ahesion to vascular endothelium (Rother et al 2005). Obviously, additional acquired or inherited risk factors may increase individual predisposition to thrombosis; genetically, the Factor V Leiden mutation and the $677 \mathrm{C}>\mathrm{T}$ methylenetetrahydrofolate reductase gene variant (as well as MTHFR polymorphisms leading to hyperhomocysteinemia) may have a relevant role, although this has not been proven so far (Nafa et al 1996). 
Table I Glycosyl-phosphatidyl inositol (GPI)-linked proteins on blood cells and their function

\begin{tabular}{|c|c|c|}
\hline Antigen & Alternative denomination & Function \\
\hline$\overline{C D I 4}$ & LPS-LPB-r & Monocyte adhesion and activation \\
\hline CDI6 & Fc $\gamma R-I I l b$ & Low affinity receptor for $\lg G$ \\
\hline CD24 & & $\mathrm{Ca}^{2+}$ flux, triggers $\mathrm{H}_{2} \mathrm{O}_{2}$ production by $\mathrm{PMN}$ \\
\hline CD48 & BLAST-I & Counter-receptor for CD2 \\
\hline CD52 & Target of Campath-I & Unknown \\
\hline CD55 & DAF & Accelerates $\mathrm{C} 3$ and $\mathrm{C} 5$ convertase decay, Cromer antigen \\
\hline CD58 & LFA-3 & Counter-receptor for $\mathrm{CD} 2$ \\
\hline CD59 & MIRL & Binds $\mathrm{C} 8 / \mathrm{C} 9$, thus inhibiting $M A C$ assembly \\
\hline CD66b & CGM6 (CEA family member) & Granulocyte adhesion/activation \\
\hline CD66c & NCA & Granulocyte adhesion/activation \\
\hline CD73 & 5'-NT & Purine/pyrimidine ribo/deoxyribonucleoside phosphorilation \\
\hline CD87 & UPAR & Urokinase receptor \\
\hline CD90 & Thy-I analog & Adhesion \\
\hline CDI08 & & Unknown \\
\hline CDI09 & Platelet Gove/b alloantigen & Unknown \\
\hline CDI57 & BST-I & enzyme; adhesion/signalling (CD38 family) \\
\hline $\mathrm{ACHE}$ & Red cell acetylcholinesterase & Enzyme, unknown substrate on RBC \\
\hline NAP & Neutrophil alkaline phosphatase & Enzyme, granulocyte function \\
\hline $\operatorname{PrPc}$ & Cellular prion protein & Unknown \\
\hline TRAIL R-3 & Trail receptor III; DcR-I & Decoy receptor for TRAIL \\
\hline ULBPs & UL- 16 binding proteins & Activating ligand for NKG2D/DAPI0 receptor \\
\hline PRV-I & Polycythemia rubra vera I & Unknown \\
\hline $\mathrm{NBI} / \mathrm{NB} 2$ & & Neutrophil antigen \\
\hline $\mathrm{JMH}$ & John Milton Hagen antigen & Red cell antigen \\
\hline \multicolumn{2}{|c|}{ Holley Gregory } & Red cell antigen \\
\hline \multicolumn{2}{|c|}{ Dombrock } & Red cell antigen \\
\hline \multicolumn{2}{|c|}{ YT } & Red cell antigen \\
\hline \multicolumn{2}{|c|}{ Platelet GP500 } & Platelet antigen \\
\hline \multicolumn{2}{|c|}{ Platelet GPI 75} & Platelet antigen \\
\hline
\end{tabular}

Abbreviations: MAC, membrane attack complex; PMN, polymorphonuclear leukocytes; RBC, red blood cells.

Looking at PNH biology in depth, we have discussed the original molecular defect in HSCs and some subsequent functional abnormalities of the progeny blood cells leading to specific clinical manifestations; however, what is the missing link and, likely, the true mystery of PNH is the reason for the expansion of GPI-deficient clones within the context of a bone marrow failure syndrome. Of note, hematopoiesis in PNH is a mosaicism of phenotypically normal HSCs and PIG- $A$ mutated HSCs; in addition, regardless of the genetic defect, hematopoiesis is impaired in almost all PNH patients, even in the presence of a broad clinical heterogeneity (Rotoli et al 1982; Maciejewski et al 1997). As mentioned above, PNH is a true disease of the HSC, as marked by the PIG-A mutation; however, the HSC defect does not entirely explain the phenotype. In fact, a series of observations suggest that the $P I G-A$ mutation is necessary but not sufficient per se to cause the disease:

(i) A few (10-50 cells per million) circulating granulocytes harboring the PNH phenotype may be demonstrated also in healthy individuals by flow cytometry, and their specific $P I G-A$ mutation may be identified by a nested PCR technique (Araten et al 1999; Hu et al 2005).

(ii) GPI-AP deficient lymphocytes were detected in lymphoma patients shortly after treatment with alemtuzumab (a monoclonal antibody that recognizes the GPI-AP CD52), and progressively disappeared after treatment withdrawal (Hertestein et al 2005).

(iii) The expansion of the aberrant PNH clone seen in PNH patients could not be reproduced in several murine models developed with the aim of creating the disease (Rosti et al 1997; Jasinski et al 2001; Keller et al 2001), even when embryonic stem cells were employed. Thus, at least in these models, the PNH clone does not seem to have any intrinsic proliferative advantage to overcome normal hematopoiesis (Jasinski et al 2001; Keller et al 2001); this is also confirmed by several data on in vitro growth of patient-derived PNH and wild-type hematopoietic progenitors (Young and Maciejewski 2000).

(iv) PNH hematopoiesis is certainly clonal, but not necessarily monoclonal. In fact, PNH hematopoiesis may in 


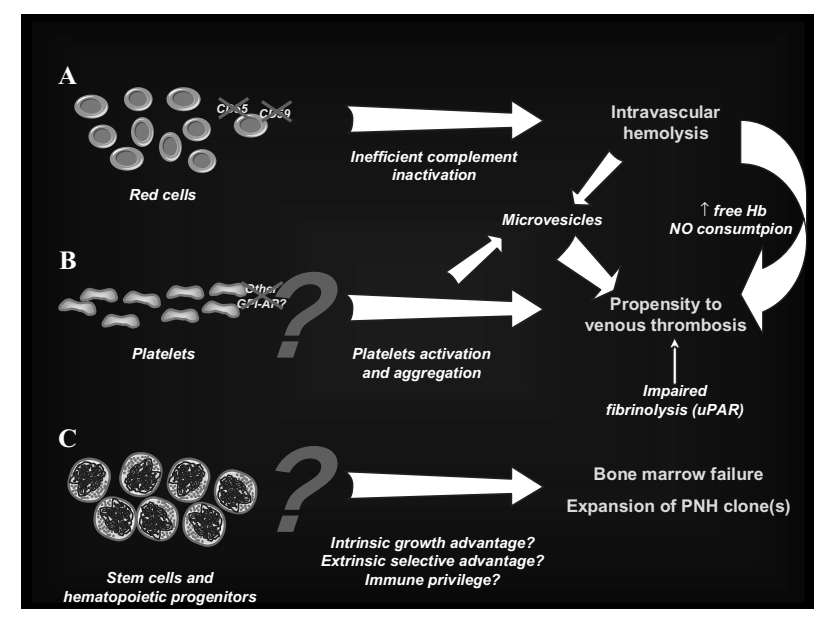

Figure 2 Effect of glycosyl-phosphatidyl inositol anchored proteins (GPI-AP) deficiency on blood cell populations. A. The lack of the complement regulators CD59 and CD55 on red blood cells (RBCs) accounts for their susceptibility to complement mediated lysis resulting in the clinical hallmark of $\mathrm{PNH}$, intravascular hemolysis. B. Deficiency of complement regulators on platelets may account for their putative propensity to activation and aggregation, possibly leading to increased clot formation and thromboembolism. Thrombophilia in PNH may also be related to NO consumption occurring in the presence of increased free $\mathrm{Hb}$ resulting from hemolysis, as well as to the impairment of the fibrinolytic system due to the lack of membrane uPAR (see text). C. The lack of anchored proteins GPI-AP has been postulated as putative cause of the expansion of the PNH clone over normal hematopoiesis; the presence of a GPI-linked immune target or a more generic reduced sensitivity to immune effector mechanisms has been hypothesized to explain the immune escape of the PNH clone, but definitive evidence is still lacking.

some cases be oligo- rather than monoclonal, as initially supported by differential susceptibility to complement lysis (Rosse et al 1966), subsequently by flow cytometry (van der Schoot et al 1990), and finally confirmed by $P I G-A$ sequencing (Endo et al 1996); this observation raised the question of whether the expansion of more clones carrying the same functional defect, but molecularly heterogeneous, is compatible with a random process, or rather may result from not stochastic processes, such as selection. The latter hypothesis may be supported by the observation that relapse of $\mathrm{PNH}$ may be sustained by clones harboring $P I G-A$ mutations which are different from those identified at diagnosis (Nafa et al 1998).

According to this background, the hypothesis of a dual pathophysiology for PNH has been developed, also known as the "relative advantage" (Figure 1B) (Rotoli and Luzzatto 1989; Luzzatto and Bessler 1996) or the "escape" theory (Dunn et al 2000). According to this theory, a mutation in the $P I G-A$ gene might be a fairly common phenomenon, with no major biological consequences, because the mutated cell has no chance of expanding in the presence of a vast majority of normal cells. In fact, no intrinsic proliferative advantage has been demonstrated in PNH hematopoietic progenitors (Araten et al 2002). However, external factors may alter this equilibrium, creating an environment permissive for the expansion of $\mathrm{PNH}$ clone(s), and possibly leading to the occurrence of a single $P I G-A$ mutated stem cell sustaining hematopoiesis even for the rest of the patient's life (Nishimura et al 2002). The nature of such extrinsic trigger may be inferred from the observation that most PNH patients also show evidence of bone marrow failure, and that several aplastic anemia (AA) patients may harbor distinct PNH clones. Thus, it is reasonable to hypothesize that pathogenic mechanisms involved in AA may also play a pivotal role in $\mathrm{PNH}$, representing the additional factor required for developing clinical PNH once a $P I G-A$ mutation has occurred (without any specific chronologic order).

Indeed, in acquired marrow failure syndromes HSCs are known to be intrinsically normal, but subject to an extrinsic damage affecting their hematopoietic function. In idiopathic AA immunological mechanisms play a pivotal role in damaging the hematopoietic compartment, leading to HSC pool consumption or functional impairment and subsequent pancytopenia. A wealth of experimental and clinic evidence supports the presence of anti-hematopoiesis immune attack, although the target antigens are still undefined, as are the mechanisms leading to the breach in immune tolerance. Unknown triggers of autoimmunity induce a cellular immune response resulting in a preferential expansion of specific $\mathrm{T}$ cell clones that may damage the hematopoietic

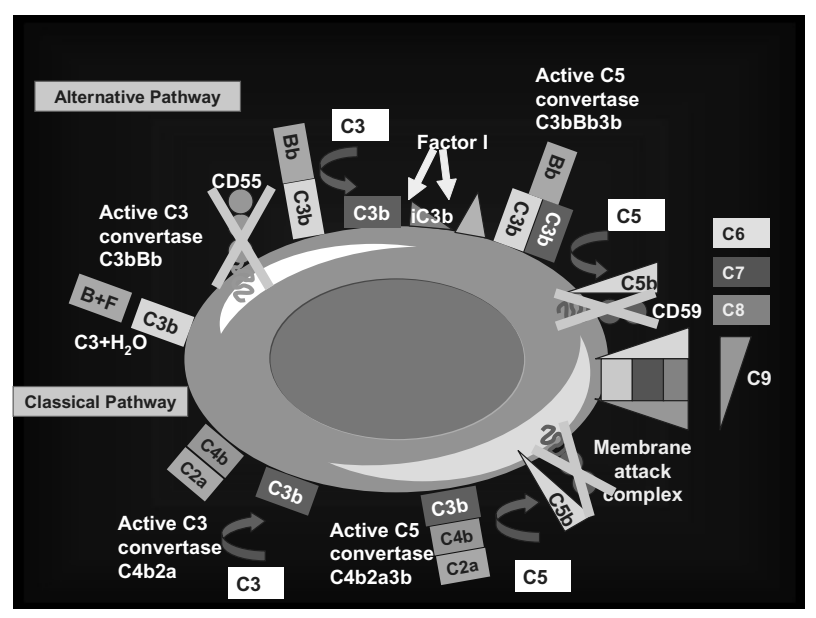

Figure 3 Mechanism of hemolysis in paroxysmal nocturnal hemoglobinuria red blood cells (PNH RBCs). The activation of both the alternative and the classical pathways of the complement cascade converge on the activation of a C5 convertase; $\mathrm{PNH}$ RBCs lack CD59, which normally inhibits the assembly of membrane attack complex (MAC), and undergo hemolysis. In addition, the early alternative pathway is dysregulated on PNH as a result of the absence of the C3 convertase inhibitor CD55; thus, $\mathrm{PNH}$ RBCs experience uncontrolled complement activation through the alternative pathway. This may explain C3 accumulation on PNH RBCs when hemolysis is blocked in presence of the terminal complement inhibitor eculizumab. 
progenitors, directly or through indirect mechanisms, such as the production of inhibitory cytokines. Stem cell damage can be mediated by cytokine-transduced inhibition (mostly type I cytokines, such as interferon- $\gamma$ and tumor necrosis factor- $\alpha$ ) (Sato et al 1997; Dufour et al 2001; Sloand et al 2002), or by direct cell-mediated killing due to cytotoxic lymphocytes (CTLs). Such mechanisms, which may attack innocent bystander cells in addition to the primary target cells, ultimately result in apoptosis, the main key mechanism of HSC damage. Circulating and marrow CTLs have been demonstrated in vivo in AA patients, and their inhibitory effect on hematopoiesis has been documented in vitro (Zoumbos et al 1995; Nakao et al 1997; Zeng et al 2001; Kook et al 2002; Risitano et al 2002; Plasilova et al 2003); recently, we have dissected this cellular immune response at the molecular level through the identification of in vivo dominant $\mathrm{T}$ cell clonotypes, which are evidence of a pathogenic antigen-driven immune response (Risitano et al 2004).

In $\mathrm{PNH}$, an antigen-driven immune response targeting the marrow tissue may be postulated, which possibly correlates with the selective expansion of the $P I G-A$ mutated clone; in fact, if the target on HSC membrane is a GPIlinked molecule, PNH HSC may escape this injury while normal HSC are killed. Evidence of immune derangement in PNH has been produced; as for AA, oligoclonality of the $T$ cell pool has been reported (Karadimitris et al 2000), and immunodominant pathogenic CTL clones may be detected in most PNH patients (Risitano et al 2002, 2004; Plasilova et al 2004; Gargiulo et al 2007), sometimes phenotypically resembling a subclinical LGL proliferation (Risitano et al 2005). In addition, according to a recent report, these effector $\mathrm{T}$ cells overexpress the activating isoforms of inhibiting superfamily receptors which elicit a powerful cytolytic activity (Poggi et al 2005).

Gene expression profiling has been employed to investigate putative differences between normal and PNH HSCs: when CD34+ cells from PNH patients were separated according to the presence or absence of GPI-AP on their surface, distinct patterns of gene expression were identified. Phenotypically normal (GPI-AP positive) CD34+ cells harbored diffuse abnormalities of their transcriptome, with over-expression of genes involved in apoptosis and immune activity, paralleling the findings seen in CD34+ cells of AA patients. By contrast, phenotypically abnormal PNH CD34+ (GPI-AP negative) showed a gene expression profiling closer to that obtained in CD34+ cells from healthy individuals (Chen et al 2005). The presence of sublethal damage in phenotypically normal HSCs but not in their $P I G-A$ mutated counterpart strongly supports the presence of an immune attack to the hematopoietic stem/progenitor cells that spares PNH cells; thus, they ultimately expand as a result of a selective pressure negatively acting on normal hematopoiesis. The "escape" of PNH cells may be interpreted in various manners. Contradictory data have been produced on a putative differential sensitivity to inhibitory stimuli between normal and PNH cells; susceptibility to apoptosis has been reported to be increased, normal, or decreased in different models. Recently, it has been shown that human cell lines carrying the $P I G-A$ mutation are less susceptible to NK-mediated killing compared to their normal counterpart (Nagakura et al 2002). In a more sophisticated model, GPI-deficient cells were not able to induce primary and secondary stimulation of both antigen-specific and alloreactive $\mathrm{T}$ cells, providing experimental support to the hypothesis that the PNH clone could inefficiently interact with the immune system (Murakami et al 2002). However, the actual mechanisms causing the escape are still elusive. They may include the absence of specific GPI-APs directly targeted by effector immune cells, or a protection due to the absence of important molecules involved in cell-cell interaction (eg, accessory molecules). Alternatively, a broader impaired sensitivity to common effector mechanisms may be hypothesized, possibly due to the lack of GPI-APs or to non-specific structural changes of the raft structure in the outer surface.

\section{Clinical features and natural history Diagnosis}

PNH has to be suspected in patients showing mild to severe anemia with moderate reticulocytosis, elevated serum lactate dehydrogenase (LDH) and possibly mild jaundice, with negative Coombs test; all these clues suggest a non-immune hemolytic anemia. The occurrence of dark urine and urinary hemosiderin, both evidence of intravascular hemolysis, strongly suggest PNH. Additional signs to be considered are the presence of mild to severe leuco-thrombocytopenia and/or a history of thromboembolic events of unknown origin, including cerebrovascular accidents. In specific conditions, PNH may be considered even in the absence of clinically evident hemolytic anemia, such as in patients with AA or those showing recurrent thromboembolic events in the absence of documented risk factors; all these patients may deserve a careful screening for PNH.

Until the eighties, the diagnosis was confirmed by the Ham test, which assesses the lysis of RBCs in acidified serum; less specific but simpler tests were the sucrose and the sugar 
water test (both based upon lysis in serum with reduced ionic strength). However, at present the diagnosis of PNH is based on flow cytometry analysis of blood cells (Parker et al 2005; Rotoli et al 2006); the high sensitivity and specificity of this analysis made the Ham and similar tests obsolete. In fact, fluorochrome-conjugated monoclonal antibodies specific to several GPI-APs expressed on the various blood cell lineages are available for routine testing; thus, simultaneous multiparameter analysis allows accurate detection of GPI-AP deficient populations, measuring their extent within each cell lineage. By this technique, one or two RBC populations with abnormal expression of GPI-APs may be shown in PNH patients: one completely lacking GPI-AP expression (type III PNH cells), and another characterized by GPI-AP faint (dim) expression (type II PNH cells). These findings match the observation initially made by Rosse (Rosse 1990), demonstrating the presence of distinct subpopulations of RBCs with different sensitivity to complement-mediated lysis; as discussed above, this may also imply that different populations may be genetically different, reflecting the expansion of two or more clones (as a result of a selective pressure for $P I G-A$ mutated HSCs). The finding of GPI-AP deficient populations may be obtained for each cell lineage, even if discrimination between type II and type III PNH cells is more difficult for white blood cells; antibodies specific for GPI-APs selectively expressed by different cell lineage may render the test more sensitive and specific. The simultaneous absence of different GPI-linked proteins on the same cells validates the specificity of the test; using this method, flow cytometry analysis detects even very small PNH clones (below 1\% of the tested cell population) (Alfinito et al 1996; Boccuni et al 2000; Richards et al 2000). More recently, the novel fluorescent reagent aerolysin, which specifically binds the GPI anchor, showed even greater sensitivity, with detection limits dropping by 1-2 logs compared with standard flow cytometry (Brodsky et al 2000; Sutherland et al 2007). Molecular studies on DNA or mRNA, aimed to identify the causative specific mutation within the $P I G$ - $A$ gene, are clinically unnecessary (even normal individuals may harbor a few PNH cells); in fact, they do not add clinically relevant data and may be even misleading, so they are now usually limited to research purposes.

\section{Classification of $\mathrm{PNH}$}

Recently, a group of experts has classified PNH into distinct clinical entities, based on the improved information coming from the use of flow cytometry. According to this classification (Parker et al 2005), three subgroups have been proposed: i. classic PNH; ii. PNH in the setting of another bone marrow disorder (eg, aplastic anemia, myelodysplastic syndrome, or myelofibrosis); iii. PNH-subclinical, in the setting of another bone marrow disorder (as above). However, this classification may be argued, and some concerns need to be addressed. There is overlap between classic PNH and $\mathrm{PNH}$ in the setting of another bone marrow disorder, given that hemolysis and some degree of bone marrow failure are hallmarks in both groups. Indeed, the presence of signs of marrow failure (ie, leukopenia, thrombocytopenia, reduced number of circulating and bone marrow colony forming cells) cannot be considered a discriminator between different groups of patients. In our opinion, some degree of marrow failure is present in all classic $\mathrm{PNH}$ patients, consistent with the pathophysiology of the disease, as described by the dual theory discussed above. Patients showing severe marrow failure may have various degrees of hemolysis, from the massive type as in classic $\mathrm{PNH}$, to the subclinical one, with all the intermediate forms; thus it is difficult to identify precise cut-offs. Similarly, some degree of morphologic abnormalities definable as dysplasia, mostly of the erythroid lineage, may be consistent with stressed hematopoiesis; thus, the difference between PNH and PNH in the context of myelodysplastic syndromes is not clear-cut. Even the presence of karyotypic abnormalities may be an epiphenomenon resulting from clonal hematopoiesis and fixation of possible mutations, rather than an indicator of the presence of a true myelodysplastic (preleukemic) clone (Araten et al 2001). Finally, PNH-subclinical refers to the presence of very small GPI-deficient clones deprived of any clinical significance; in this context, the term PNH itself is misleading and should not be used, since none of the clinical manifestations of this condition are present. The term "acquired GPI-AP deficiency" seems to be more appropriate, and might be extended to the entire classification (possibly including other acquired GPI-AP deficiency, such as those emerging within lymphocyte subsets after treatment by alemtuzumab) (Hertenstein et al 1995). In addition, this definition also better applies to the rare patients showing GPI-AP deficient neutrophils and monocytes, with no PNH erythrocytes; such patients, who possibly reflect a $P I G-A$ mutation occurring in committed hematopoietic progenitors rather than in a multipotent HSC, remain often undiagnosed and are excluded from the proposed classification.

\section{Natural history}

As recalled above, $\mathrm{PNH}$ is dominated by the clinical triad of chronic intravascular haemolytic anemia, propensity 
to develop thromboembolic events and stigmata of bone marrow failure. While intravascular hemolysis is constant in all typical PNH patients, thrombosis and cytopenia have a variable prevalence within the $\mathrm{PNH}$ population and may be undetectable in single patients. This is mostly true for thromboembolic events, which are erratic and unpredictable, and may not affect all individuals, although recurrence is frequent after the first episode (Hillmen et al 1995; Socie et al 1996).

Hemolysis is present in all PNH patients, but anemia occurs with variable severity; this results from the degree of complement activation and subsequent hemolysis, as well as from the capacity of compensatory erythropoiesis, which depends in turn on the efficiency of the residual marrow function (indicated by reticulocyte count). Indeed, the size of the PNH clone within the total RBCs and the type of PIG- $A$ molecular lesion accounting for the different sensitivity to complement-mediated lysis (ie, type II and type III PNH RBCs) shape the clinical picture, with some patients having an almost normal hemoglobin level and others requiring massive transfusional support. Exacerbations of hemolysis are typical, and account for the term paroxysmal nocturnal hemoglobinuria; they are unpredictable in most cases, although some patients experience quite regular crises. Paroxysms likely reflect transient complement activation above the physiological low-level (which is due to the alternative pathway), and are often associated with external triggers such as infections and inflammatory responses, which massively activate both the classical and alternative complement pathways. Beside the paroxysms, anemia may significantly vary during the clinical course of individual patients, with cases suddenly worsening and others unexpectedly improving in the absence of any known reason; anecdotal cases showing spontaneous remission of the disease have also been reported (Hillmen et al 1995; Socie et al 1996; Peffault de Latour et al 2006). Signs of intravascular hemolysis are jaundice, hemoglobinuria, increased LDH, and increased unconjugated bilirubinemia; the symptoms are those common to other types of hemolytic anemia, such as asthenia, fatigue to the extent of lethargy, with significant quality of life impairment. In addition, some specific symptoms have been described, mostly in association with paroxysms, including dysphagia, painful abdominal crises and erectile dysfunction; these symptoms have been linked to smooth muscle dystonia possibly resulting from local nitric oxide (NO) deficiency subsequent to NO consumption by excess free hemoglobin (Rosse 2000; Moyo et al 2004; Rother et al 2005). All these symptoms significantly affect the well-being of PNH patients, even if they are often underestimated by physicians because they are not life-threatening.

Cytopenias are frequent, and may be anything from mild to severe, depending on the underlying bone marrow defect; some patients may develop true aplastic anemia during the course of the disease, and may require specific treatment. The consequences of cytopenia include increased risk of infection and hemorrhage; their management depends on the severity of the cytopenia and on the clinical picture, paralleling that of aplastic patients. In addition, a specific infectious risk may be present as a result of impaired function of $\mathrm{PNH}$ neutrophils and monocytes, mostly affecting microorganism destruction through the radical oxygen species pathway (Cacciapuoti et al 2007).

Thrombosis is the most life-threatening clinical manifestation. It usually affects veins, and occurs in sites unusual for patients with other types of thrombophilia; typical sites are the suprahepatic veins, leading to the Budd-Chiari syndrome, mesenteric veins, the splenic vein, and cerebral veins. The incidence of thrombosis seems to be different according to ethnicity, with Asian patients showing lower risk than Europeans or Americans, or particularly Afro-Americans and Hispanics (Nishimura et al 2004). Maybe this results from the multifactorial pathophysiology of thrombosis in $\mathrm{PNH}$ as previously discussed, and from the possible contribution of genetic factors predisposing to thrombosis. In various observations thromboses have been reported associated with massive intravascular hemolysis and larger WBC PNH clones (Hall et al 2003; Moyo et al 2004); even if this statement has to be confirmed, it has to be remarked that even patients with small clones have a significant thrombosis risk compared to healthy individuals (Hall et al 2003). Thromboembolic events may significantly affect survival; as a whole, thrombosis may affect half of PNH patients, accounting for at least one third of mortality.

$\mathrm{PNH}$ is a clonal but non-malignant disease; even if progression to acute leukemia has been occasionally reported (sometimes leading to overestimation as a result of preferential reporting), it has been estimated in the range of $2 \%-3 \%$, similar to that reported for AA patients. $\mathrm{PNH}$ is a very rare disease; information on large series are few and somewhat dated; however, the estimated median survival may be over 10 years, with a quarter of patients surviving longer than 25 years (Socié et al 1996; Hillmen et al 2005; Peffault de Latour 2006). These data may have a bias due to a negative selection of patients presenting with early complications (eg, those with lethal thrombosis), and do not consider changes 
in the management of $\mathrm{PNH}$, including transplant procedures and, more recently, eculizumab treatment. As discussed, the major causes of death are thrombosis, followed by infectious complications and, lastly, hemorrhage; thus, an aplastic presentation significantly affects the prognosis. Notwithstanding the relatively long survival, the course of the disease may be stormy, with frequent hospitalizations, possible comorbidities, and subsequent poor quality of life.

\section{Management: the pre-eculizumab era \\ Supportive treatment}

The treatment of $\mathrm{PNH}$ is mainly supportive, aiming to control the clinical manifestations of the disease. Thus, it may be divided into management of hemolysis and anemia, management of thrombophilia, and management of bone marrow failure.

\section{Management of hemolysis and anemia}

Hemolysis and the subsequent anemia are the hallmarks of $\mathrm{PNH}$, and usually require specific treatment. Unfortunately, until the new millennium there were no clinically satisfactory options for controlling hemolysis. Steroids were broadly used as chronic administration or for acute hemolytic crises. Indeed, some investigators claim that steroids are useful in controlling chronic complement-mediated hemolysis (Issaragrisil et al 1987; Bourantas 1994) and, even more, paroxysmal crises, likely interfering with complement activation and/or underlying conditions triggering the complement cascade; however, so far no conclusive data or convincing mechanisms of action have been provided. Clinically, steroids seem to ameliorate patient well-being even in the absence of complete control of hemolysis (Parker et al 2005); in particular, they may be effective in improving symptoms associated with paroxysmal crises, such as dysphagia and abdominal pain (possibly as a result of less severe crises). However, since the long-term toxicity of chronic steroidal therapy, in the range of $0.5-1 \mathrm{mg} / \mathrm{kg}$, advises against its systematic use in PNH patients, it is currently not recommended.

Androgens have been utilized, too, with limited benefit (Harrington et al 1997); however, given their potential utility in stimulating erythropoiesis and megakaryocytopoiesis (Katayama et al 2001), they are primarily indicated in the presence of signs of marrow impairment rather than to control hemolysis. Once again, a risk-benefit evaluation should be specifically made for all individual cases, given that liver toxicity, virilizing action, and other side effects have to be considered; in addition, some concerns about a potential increase in the Budd-Chiari syndrome have been raised by some physicians (Parker et al 2005). A few patients in our series benefited from danazol, a non-virilizing anabolic steroid, given at doses of 400-600 mg/day.

Thus, given the impossibility of blocking intravascular hemolysis, the main strategy to control anemia was transfusions. RBC transfusions are given to $\mathrm{PNH}$ patients according to their hemoglobin level, which may be highly variable even in the same patient; as for other anemic patients, transfusions should be administered to maintain hemoglobin level above $8 \mathrm{gm} / \mathrm{dL}$. Transfusions transiently improve anemia-related symptoms; however, refractoriness to transfusion may develop, and is considered a severe complication requiring more aggressive treatment - namely stem cell transplantation. In contrast to other transfusion-dependent patients, those with PNH have a low risk of iron overload, given the massive iron loss due to hemoglobinuria and hemosiderinuria. Thus, strategies for iron chelation are not necessary; in fact these patients may sometimes even require iron supplementation to sustain the compensatory erythropoiesis (Rosse 1982). Similarly, vitamin B12 and folate supplementation are usually indicated to sustain the enhanced erythropoiesis (Rosse 1982); furthermore, endogenous erythropoiesis may also be increased by recombinant erythropoietin, mostly in cases with inadequate production (Stebler et al 1990; Bourantas 1994; McMullin et al 1996). Paradoxically, all these strategies, including iron replacement, may lead to increased hemolysis as a result of increased PNH hematopoiesis, contrary to what is seen following suppression of erythropoiesis by transfusions.

\section{Management of thrombophilia}

The management of the propensity to develop thrombosis is a hot issue in $\mathrm{PNH}$, given that this complication represents the first cause of death. Unfortunately, there are no controlled prospective clinical trials on primary and secondary thrombosis prophylaxis, nor on acute treatment of the event. The issue of primary prophylaxis is controversial, and no consensus exists; some physicians advocate the use of warfarin for all newly diagnosed $\mathrm{PNH}$ patients, while others do not use any prophylaxis. Both approaches are reasonable, given the unpredictability of thromboembolic events and the lack of evidence supporting any of these strategies; possible benefits are counterbalanced by the risk of hemorrhage from warfarin therapy, which may be considerable in $\mathrm{PNH}$ patients with low platelet count. With the recent attempt to identify patients at higher risk of thrombosis, a reasonable compromise may be the adoption of prophylaxis for patients 
with WBC PNH clones larger than $50 \%$, or those with additional genetic risk for thrombosis, including ethnicity. Using such and approach, Hall and colleagues (Hall et al 2003) demonstrated a very low incidence of thromboembolic events compared with historical controls; however, these data need to be confirmed in larger series and possibly in prospective randomized trials that include alternative, more manageable, agents such as antiplatelet drugs or the new thrombin inhibitors.

The opinion on secondary prophylaxis is less controversial, with a general agreement that all PNH patients experiencing any thromboembolic event should remain life-long on anticoagulants; however, even in this setting, no consensus exists on the best strategy. Low-molecular-weight heparin, as well as warfarin at different therapeutic ranges, are both utilized, with some physicians even considering the addition of anti-platelet agents. However, despite this extended prophylaxis, recurrence of thrombosis (either as new events or progression of the existing ones) is frequent and affects the survival of PNH patients (Audebert et al 2005). Moreover, life-threatening hemorrhagic events are quite frequent in this cohort of patients, mostly when concomitant thrombocytopenia is present (Hall et al 2003; Moyo et al 2004).

Finally, the management of an acute thromboembolic disease may require an intensive therapy similar to that for myocardial infarct; fibrinolytic therapies using tissue plasminogen activator have been exploited, showing efficient clearance of the thrombus in individual cases (McCullin et al 1994; Hauser et al 2003).

\section{Management of marrow failure}

The management of marrow failure in PNH patients is the same as that for AA patients. Indeed, in addition to supportive strategies such as anti-infectious, anti-thrombotic, and anti-hemorrhagic prophylaxis and/or treatment, etiologic therapies can also be attempted. According to the pathogenic mechanisms and the dual hypothesis described above, an immune-mediated inhibition of hematopoiesis is postulated in $\mathrm{PNH}$, similar to that demonstrated in AA. Thus, immunosuppressive strategies have been reasonably attempted in PNH patients, using regimens with antilymphocyte or antithymocyte globulin associated with high dose prednisone and cyclosporin A, as those recommended in AA (Tichelli et al 1992); the results have been quite variable (Sanchez-Valle et al 1993), even if extensive studies are still lacking. Newer immunosuppressive agents such as the anti-CD52 monoclonal antibody alemtuzumab may be an alternative candidate as immunosuppressant; in this setting, there is no concern about the potential risk of selecting for PNH hematopoiesis, given that the GPI-linked CD52 is not expressed on HSCs.

So, after 3 decades of efforts the conclusion is that no therapy adequate to the patients' medical needs had been developed, and most patients had to face every day the symptoms of their disease.

\section{Curative strategies}

The only curative approach for $\mathrm{PNH}$ is allogeneic hematopoietic stem cell transplantation (SCT); alternative strategies include cell therapy (insertion of molecules on the outer surface of blood cells) (Hill et al 2006) and gene therapy (insertion of a functional $P I G$ - $A$ gene in early hematopoietic progenitors), but all experimental efforts have so far been unfruitful and seem to be far away from clinical applications. In addition, if the escape theory is correct, the latter approach may not result in clinical benefit in $\mathrm{PNH}$, since a repair of the damaged cell should result in cell destruction, as is believed to occur for normal hematopoiesis in $\mathrm{PNH}$ patients.

SCT has been exploited since the late 1980s, with very positive long-term results; however, SCT is burdened by significant early transplant-related mortality. Most reports in the literature refer to single cases or small series from single institutions (Bemba et al 1999; Saso et al 1999; Raiola et al 2000), while large prospective studies are lacking. An overview has been recently published by Parker et al (Parker et al 2005), who collected data from 67 patients transplanted from different types of donors (syngeneic, sibling, or HLAidentical unrelated) and using different types of conditioning (myeloablative or reduced intensity). Data from the entire group showed a $75 \%$ long-term survival, which is higher than that reported in a majority of individual series, likely as a result of a reporting bias. A recent survey from the Italian Transplant Group (GITMO) on 23 PNH patients transplanted between 1988 and 2006 showed a 6-month treatment-related mortality of $26 \%$ and an overall survival of $70 \%$, with a median follow-up of 107 months (Santarone et al 2008). A similar retrospective study is currently ongoing within the European Blood and Marrow Transplantation (EBMT) group, and promises to provide useful information for evidence-based decision making; preliminary results confirm an overall survival in the range of $55 \%-65 \%$, with older age and acute GvHD significantly affecting the outcome, and apparently comparable results between sibling and unrelated transplants.

Given these results, guidelines for SCT in PNH are hard to define; the procedure should be restricted to young 
patients with a suitable HLA matched donor, even if the improvement in transplant procedures may extend SCT even to older recipients, once the indication is established. A clear indication may be an underlying bone marrow failure unresponsive to immunosuppression; thrombosis and refractoriness to transfusion may be additional indications, even if they should be re-evaluated in the present era of biological agents, namely eculizumab. Specific procedures to achieve the best outcome will, we hope, result from the ongoing retrospective and prospective studies, mostly investigating whether the new reduced-intensity conditioning regimens may further reduce transplant-related mortality as well as long-term complications.

\section{Management: the eculizumab era}

\section{Mode of action and pharmacokinetics}

Eculizumab (h5G1.1-mAb, Soliris ${ }^{\circledR}$, Alexion Pharmaceuticals) is a humanized monoclonal antibody (mAb) (Figure 4) derived from the murine anti-human $\mathrm{C} 5 \mathrm{mAb}$ (Rother et al 2007). It specifically binds the terminal complement fraction 5, thereby inhibiting the cleavage to $\mathrm{C} 5 \mathrm{a}$ and $\mathrm{C} 5 \mathrm{~b}$ (Figure 5) (Matis and Rollins 1995), thus blocking the formation of the membrane attack complex (MAC), which is the terminal effector mechanism leading to intravascular hemolysis of PNH RBCs. In addition, eculizumab prevents the release of pro-inflammatory mediators resulting from C5a cleavage. The blockade of the complement cascade at the level of C5 does not affect early complement components (such as C3 and its cleavage products), preserving pivotal functions leading to clearance of immune complexes and microorganisms (Matis and Rollins 1995). Eculizumab was initially investigated in patients suffering from rheumatoid arthritis, psoriasis, systemic lupus erythematosus, dermatomyositis, and idiopathic membranous glomerulopathy; however, PNH appeared the best candidate disease to benefit from eculizumab treatment. In fact, the lack of CD59 on red cell membranes accounts for uncontrolled C5b-9 (MAC) assembly, finally resulting in intravascular hemolysis (Figure 3); theoretically, eculizumab may compensate for the absence of CD59 blocking the MAC formation, thus rendering irrelevant the absence of CD59 on PNH RBCs (Figure 6).

At a more detailed analysis of the properties of eculizumab, it appears that the antibody has a human kappa light chain constant region, while the heavy constant region results from the combination of $\mathrm{IgG} 2 \mathrm{CH} 1$ and $\mathrm{IgG} 4 \mathrm{CH} 2$ and $\mathrm{CH} 3$ domains; thus eculizumab does not activate the complement by itself. The specificity for human $\mathrm{C} 5$ is quite stringent, as demonstrated

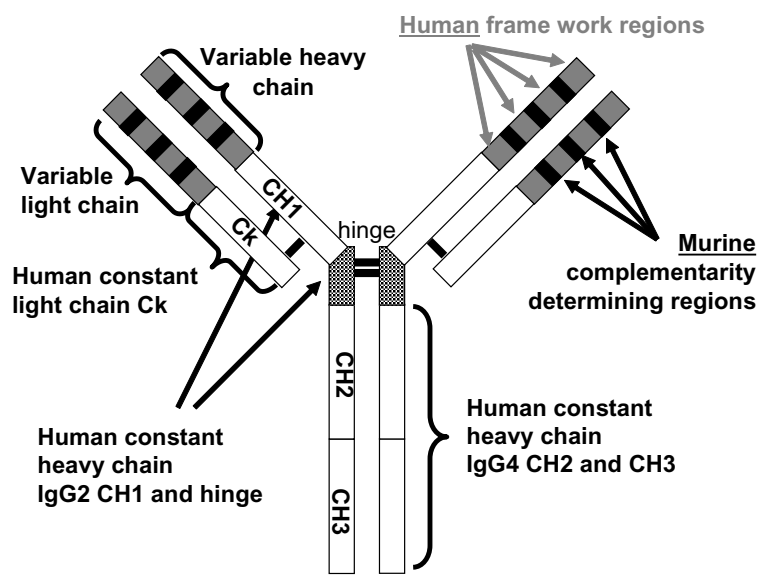

Figure 4 Eculizumab: the molecule. The structure of the anti-complement 5 humanized monoclonal antibody eculizumab (h5GI.I mAb).

by the almost total absence of species cross-reactivity. Pharmacodynamic (PD) and pharmacokinetic (PK) profiles of eculizumab have been assessed by a serum complement hemolysis in vitro assay and an ELISA analysis of serum concentration, using different dosing schedules. Hemolysis was considered inhibited if lysis of antibody-sensitized chicken erythrocytes after sample serum incubation was $<20 \%$ of that with normal serum; eculizumab through levels above $35 \mu \mathrm{g} / \mathrm{mL}$ were demonstrated to saturate the $\mathrm{C} 5$ target and to block hemolysis in more than $80 \%$ of samples. Several loading doses were evaluated, and a fixed $600 \mathrm{mg}$ weekly dose was shown to be effective in achieving pharmacodynamically effective concentrations of $>35 \mu \mathrm{g} / \mathrm{mL}$; the fixed dose was exploited after demonstrating that the $\mathrm{PK}$ profile did not correlate with weight or other patient-specific factors. The PK profile demonstrated

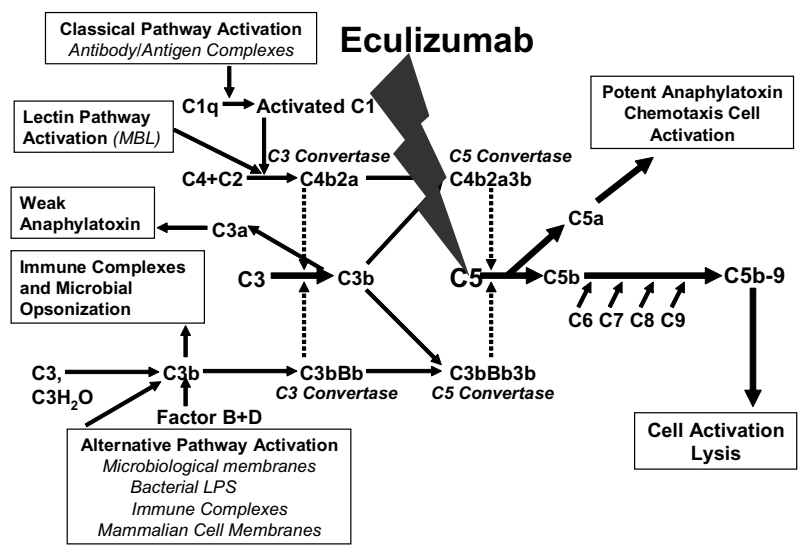

Figure $\mathbf{5}$ The complement cascade and the $\mathrm{C} 5$ blockade by eculizumab. Eculizumab blocks the cleavage of $\mathrm{C} 5$ to $\mathrm{C} 5 \mathrm{a}$ and $\mathrm{C} 5 \mathrm{~b}$; all earlier steps of the complement cascade are preserved, including C3 cleavage. 


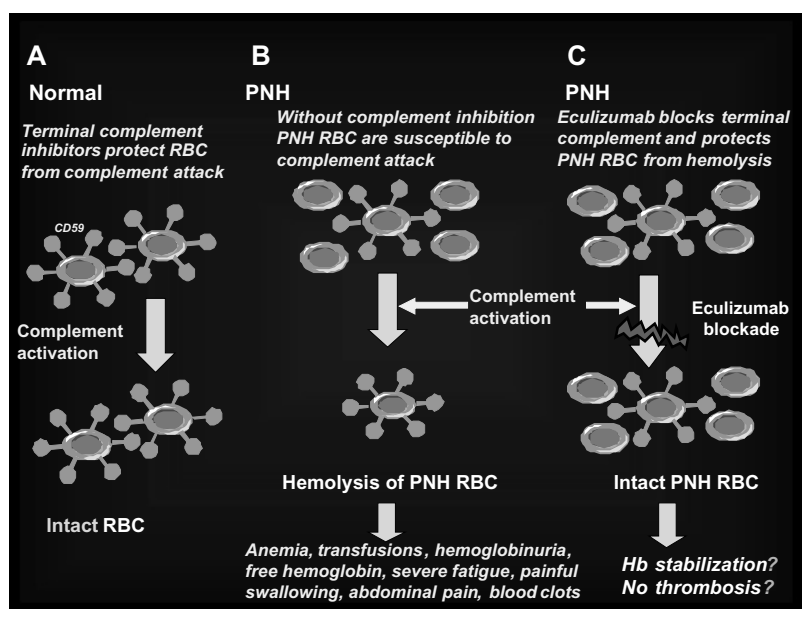

Figure 6 The rationale for eculizumab in paroxysmal nocturnal hemoglobinuria (PNH). A. Normal red blood cells (RBCs) are protected from complement attack by CD59. B. PNH RBCs are susceptible to complement attack due to lack of CD59, resulting in hemolysis and consequent clinical manifestations. C. Eculizumab blocks the complement cascade inhibiting MAC formation; thus, even upon complement activation, PNH RBCs are protected from hemolysis, thus resulting in hemoglobin stabilization and reduction of thromboembolisms.

a non-linear behavior; further complex mathematical models describing PK parameters (ie, an equation relating the volume of the central compartment to the amount of drug in that compartment) yielded an estimated half-life of 271 hours, with the onset of steady-state concentrations predicted to be reached after 57 days of therapy. Eculizumab is administered intravenously, thus its bioavailability is $100 \%$. The drug is distributed within vascular and extracellular spaces as other endogenous antibodies; the effects of non-C5 plasma proteins on eculizumab distribution have not been determined. The elimination of eculizumab is expected to involve uptake into endothelial pinocytotic vesicles containing Fc receptors and other mechanisms active in native antibody recycling; given its size, elimination through the kidneys, the liver, the lungs, and the gastrointestinal system is not expected (and even experimentally proven for the kidneys). Based on these findings, eculizumab therapy was designed to rapidly reach PD levels using an induction regimen, followed by a maintenance dosing schedule to avoid concentration drop below $35 \mu \mathrm{g} / \mathrm{mL}$. In all PNH studies eculizumab has been administered as 4-weekly doses of $600 \mathrm{mg}$ (induction regimen), followed by $900 \mathrm{mg}$ doses every other week (maintenance regimen), starting 1 week after induction (week 5); this is the standard schedule, recently approved by the FDA for the treatment of PNH.

\section{Efficacy}

Eculizumab has been evaluated in a number of clinical trials enrolling PNH patients with hemolytic disease and transfusion dependence; overall, almost 200 patients have been enrolled. The first open-label pilot study included 11 patients and provided the initial evidence of efficacy, proving the principle that blocking $\mathrm{C} 5$ may reduce hemolysis in PNH patients (Hillmen et al 2004; Hill et al 2005a). Then, the definitive evidence of efficacy was provided by 2 large multi-center trials, the TRIUMPH and the SHEPHERD studies.

The TRIUMPH study (Hillmen et al 2006) was a doubleblind, placebo-controlled study which randomized 87 patients to receive intravenously either placebo or eculizumab at 4-weekly doses of $600 \mathrm{mg}$ (induction period), followed by $900 \mathrm{mg}$ every other week (maintenance period). Inclusion criteria were transfusion-dependent $\mathrm{PNH}$ patients with no clinically significant marrow failure (no neutropenia or thrombocytopenia); the treatment period was 6 months. Eculizumab was able to inhibit in vivo the intravascular hemolysis of PNH patients, as confirmed by rapid and sustained reduction of LDH levels, measured as area under the curve, in patients receiving the drug compared with the control group. Eculizumab was more effective than placebo in improving anemia, with hemoglobin stabilization in $48.8 \%$ of patients versus $0 \%$ in the placebo group. As a result, $51.2 \%$ of eculizumab-treated patients no longer required transfusions compared with $0 \%$ of those receiving placebo. As a group, the median number of red cell units transfused was 0 in the eculizumab group and 10 in the placebo group, globally indicating a significant reduction in transfusion requirement; even patients who still required transfusions while on eculizumab showed a significantly reduced need for transfusion than before treatment. The amelioration of anemia translated into a significant improvement of fatigue and quality of life (see below).

These data were confirmed in the subsequent SHEPHERD trial (Brodsky et al 2008), an open-label study designed to test eculizumab in a broader PNH population, which included patients with minimal transfusion requirement and even those with thrombocytopenia. This international study enrolled 97 patients, who received a 52-week treatment; globally, the patients showed a significant blockade of hemolysis compared with the pre-treatment period, as assessed by an $87 \%$ reduction of LDH level. As predicted, the reduced hemolysis improved the condition of anemia, to the extent that $51 \%$ of patients did not require transfusion.

Patients originally enrolled in the TRIUMPH and the SHEPHERD studies joined the Extension study, an ongoing non-placebo-controlled trial involving patients coming from any of the previous eculizumab-based trials (including the 
initial pilot study). The Extension study was mostly designed to assess the effect of long-term treatment in terms of both efficacy and safety; however, among the endpoints there was the prospective evaluation of thromboembolic events (TE) in comparison to that of the pre-treatment period. Such endpoint has been recently evaluated in an ad interim analysis, and data have been recently published; the analysis included 195 patients who had been exposed to eculizumab (Hillmen et al 2007). The TE rate in patients on eculizumab was $1.07 / 100$ patient-years, which represents an $87 \%$ reduction compared with that inferred from the pre-treatment period $(7.37 / 100$ patient-years). Such a reduction seemed even more striking when the pre-treatment observation period was limited to the 12 months before starting eculizumab. The protection from thrombosis conferred by eculizumab in high risk patient is confirmed by data in patients on antithrombotics because of a previous TE event: eculizumab associated with antithrombotics resulted in a $0.62 / 100$ patient-years TE rate, which is significantly lower than that on antithrombotics alone, before eculizumab treatment (10.61/100 patient-years). Thus, eculizumab seems to reduce thrombosis in $\mathrm{PNH}$ patients, even in patient cohorts where common antithrombotics usually fail to show any efficacy.

Globally, the clinical efficacy of eculizumab in PNH patients consists of a rapid and sustained reduction of intravascular hemolysis, leading to increased hemoglobin level, and to reduced need - or not need at all - for transfusions; in addition, all symptoms related to hemolysis, such as abdominal pain and dysphagia, completely remitted (Hill et al 2005b). Furthermore, a significant reduction in the TE risk emerges, which may likely translate into increased life-expectancy. Such benefits were demonstrated to be maintained in long-term treatment, as no reduced efficacy has been documented even in patients receiving the drug for 3 years or longer.

\section{Safety and tolerability}

The safety profile of eculizumab was assessed in the 4 studies involving PNH patients, as well as on 11 studies utilizing eculizumab for different indications; the cumulative exposure was 147.44 and 492.20 patient-years in the two populations, respectively. Three deaths were reported in the PNH studies, all considered unrelated to the investigational drug; fatal events were related to the underlying disease in 2 cases ( 1 cerebral vascular accident and 1 progression to chronic myelomonocytic leukemia) and to an unrelated accident in the third (cerebral herniation). The main concern was a putative increased risk of infection, mostly by encapsulated bacteria, namely Neisseria spp. A case of meningitis by Neisseria meningitidis was initially described within the non-PNH cohort; since then, all patients exposed to eculizumab are vaccinated against Neisseria meningitidis using a polyvalent vaccine. In addition, all patients received a warning on meningitis and infectious symptoms, as well as a rescue antibiotic prescription. No case of meningitis has been documented among the $195 \mathrm{PNH}$ patients receiving eculizumab; however, 3 patients developed a Neisseria meningitidis infection, with sepsis in 2 cases. None of these patients developed meningitis or other complications, and all recovered promptly as a result of early diagnosis and treatment. Furthermore, at least 2 of the 3 cases seemed to be due to the Neisseria meningitidis groups, which are not covered by the prescribed vaccine. The incidence of serious adverse events was similar in eculizumab-treated patients and in those receiving the placebo within the TRIUMPH trial; furthermore, none of the serious adverse events was considered as possibly, probably, or definitely related to eculizumab. The overall rate of infectious events did not increase compared with the placebo group; however, herpes simplex and some other site-specific infections (nasopharyngitis, upper respiratory tract infection, urinary tract infection, and sinusitis) appeared to be more frequent within the eculizumab-treated population. However, in all cases the intensity was mild and the clinical resolution prompt.

Globally, the most common adverse events were headache, followed by pyrexia, nasopharyngitis, herpes simplex, nausea, fatigue, and myalgia; all these events were generally mild and resolved quickly. No discontinuation due to side effects of eculizumab was observed. There was some concern about possibly severe hemolysis episodes following withdrawal or non-compliance with the eculizumab dosage regimen; however, there was no evidence of lifethreatening or serious hemolysis, even in the few patients who discontinued treatment and progressively returned to their pre-treatment levels of hemolysis. The occurrence of immunogenity was assessed, and was demonstrated to be very infrequent, if present at all, and without consequence on drug efficacy. Treatment was discontinued in 3 patients due to pregnancy, as per protocol; of these, the two with adequate follow up gave birth to a normal baby, with no maternal complications.

In summary, a wide variety of data generated from studies of patients with $\mathrm{PNH}$, supported by safety data from studies of patients diagnosed with other diseases, demonstrate that treatment with eculizumab is safe and well-tolerated for the treatment of PNH. Long-term treatment has not 
demonstrated deviation from this safety profile; however, anti-meningococcal vaccination and warning for symptoms of meningitis remain mandatory.

\section{Patient-focused perspectives}

Patient-reported outcomes were systematically assessed in all eculizumab-based studies (Hillmen et al 2006; Hillmen et al 2007; Brodsky et al 2008); fatigue is a common symptom of PNH patients, and significantly affects their quality of life. In addition, painful and disabling hemolysis-specific symptoms may be present, finally resulting in global health status and patient functioning impairment (Hill et al 2005b). Eculizumab treatment was associated with significant improvement in fatigue, as measured by both the FACIT and the EORTC QLQ-C30 fatigue scales. In addition, eculizumab-treated patients reported a significant improvement in a number of EORTC QLQ-C30 subscales, including global health status, role, social, cognitive, physical and emotional functioning, pain, dyspnea, and appetite loss. This unequivocally demonstrated that eculizumab improves the quality of life and the well-being of PNH patients improving both anemia and anemia-related symptoms, as well as other disease-related symptoms. As a result, patient's acceptance of the treatment is enthusiastic, even if the treatment schedule requires frequent and possibly tiresome journeys and hospitalizations, usually in day hospital, to receive the drug.

\section{Future directions}

Eculizuamb was effective in controlling intravascular hemolysis in all PNH patients, as demonstrated by their LDH levels, with the exception of a few patients who experienced breakthrough hemolysis on eculizumab with the standard 14-day schedule. These patients usually show recurrence of hemolysis toward the end of the interval, ie, 1 or 2 days before receiving the following dose, and may be rescued by shortening to 12 days the interval between administrations. Beside this PK exception, all patients on eculizumab show a complete and sustained block of intravascular hemolysis; however, some of them do not achieve normalization of their hemoglobin level and show persistent high reticulocyte count and increased serum bilirubin in presence of a normal marrow function, and even after stimulation by erythropoietin (Hill et al 2007b). Thus, residual low-titer hemolysis can be suspected in most PNH patients on eculizumab (Hillmen et al 2004); the hemolysis may be due to pathophysiological mechanisms other than those so far described in $\mathrm{PNH}$ (Rosenfeld et al 1985). Based on personal ongoing investigation (Risitano et al 2006), we suggest that eculizumab may induce substantial changes in PNH biology, leading to the possible transformation of a disease with typical intravascular hemolysis into a new one characterized by prevalent extravascular hemolysis. As discussed above, eculizumab blocks C5 cleavage and subsequent intravascular hemolysis, but does not affect $\mathrm{C} 3$ deposition and cleavage on RBC membrane, which is not controlled given the absence of CD55 (Figure 3). Thus the accumulation of $\mathrm{C} 3$ allowed by the increased $\mathrm{PNH}$ RBC survival on eculizumab treatment may lead to unexpected extravascular hemolysis in the reticulo-endothelial system via complement receptors. This is confirmed by preliminary data from Italian patients, showing that in vivo RBC survival, as assessed by 51-Cr study, remains reduced in $\mathrm{PNH}$ patients on eculizumab, who have no evidence of residual intravascular hemolysis (Risitano et al 2007). In addition, $\mathrm{C} 3$ coating was demonstrated by direct anti-globulin test (C3d only) and flow cytometry in all $\mathrm{PNH}$ patients on eculizumab; these harbor a characteristic C3-positive $\mathrm{RBC}$ population with the $\mathrm{PNH}$ phenotype, in addition to the two expected populations of $\mathrm{C} 3$-negative phenotypically normal RBCs and C3-negative PNH RBCs. These findings were not found in untreated $\mathrm{PNH}$ patients, who only showed the two latter RBC populations (Risitano et al 2007). This intriguing new mechanism of disease may become apparent only upon eculizumab treatment, and is currently under investigation to assess its actual importance in vivo and explore future clinical implications, namely possible strategies to optimize the clinical efficacy of eculizumab.

\section{Conclusion}

Even if PNH is a rare condition, it represents an intriguing model of disease and has attracted the efforts of several generations of investigators in the last three decades. While the '80s unraveled the GPI-anchor and the functional defect of the PNH clone, and the 1990s revealed the $P I G-A$ gene and its role in the pathophysiology of the disease, this new century is finally bringing forth innovative therapeutic approaches. Mechanisms of disease in PNH have been almost completely elucidated in recent years and now, in the era of biological agents, have led to the development of targeted therapy through the terminal complement inhibitor eculizumab. Eculizumab showed great efficacy in blocking complement-mediated intravascular hemolysis in $\mathrm{PNH}$ patients, leading to substantial improvements in anemia, fatigue, hemolysis-related symptoms and quality of life. In addition, preliminary data suggest that eculizumab may significantly reduce the risk of thrombosis in PNH patients, likely as a result of controlling free hemoglobin level and 


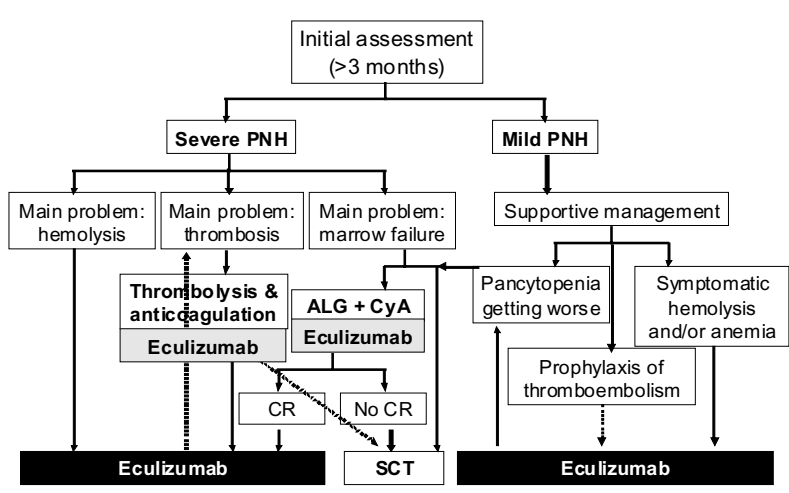

Figure 7 Algorithm of the clinical management of paroxysmal nocturnal hemoglobinuria (PNH) in the eculizumab era. Once $\mathrm{PNH}$ is confirmed, all patients suffering from its severe form should receive eculizumab to control intravascular hemolysis; in case of concomitant thromboembolic manifestations an appropriate anticoagulant and/or antithrombotic therapy should be added. In the presence of concomitant severe marrow failure, the indication of an immunosuppressive regimen should be discussed, and programmed possibly before eculizumab treatment (no data on concomitant therapy are available so far). Patients suffering from mild $\mathrm{PNH}$ should receive supportive therapy according to the main clinical manifestations: symptomatic hemolysis and/or anemia should be treated by eculizumab, signs of marrow failure may require immunosuppression. Even in this subset the use of eculizumab is reasonable as prophylaxis of thromboembolic events. Indications to stem cell transplantation remain severe marrow failure not responding to immunosuppression (or even as first line in young patients, if a low risk transplant procedure is possible) and possibly refractory thromboembolic disease (but the risk-benefit ratio in comparison to eculizumab has to be demonstrated in this setting).

inhibiting the subsequent $\mathrm{NO}$ depletion. Thus, the new millennium of PNH starts with the first treatment able to specifically control most symptoms of the disease, and likely reduce the risk of life-threatening complications, leading to a substantial change in the algorithm of the clinical management of PNH patients (Figure 7). Whether and how this new drug will affect the biology of $\mathrm{PNH}$, possibly changing the paradigm of known pathogenic mechanisms, and, more importantly, affecting patient survival are the new challenge for the next few years.

\section{Disclosures}

$\mathrm{AMR}$ and $\mathrm{BR}$ have received research grants and lecture fees from Alexion Pharmaceuticals; they have also served as consultants and on advisory committees for the same company.

\section{References}

Alfinito F, Del Vecchio L, Rocco S, et al. 1996. Blood cell flow cytometry in paroxysmal nocturnal hemoglobinuria: a tool for measuring the extent of the PNH clone. Leukemia, 10:1326-30.

Almeida AM, Murakami Y, Baker A, et al. 2007. Targeted therapy for inherited GPI deficiency. $N$ Engl J Med, 356:1641-7.

Almeida AM, Murakami Y, Layton DM, et al. 2006. Hypomorphic promoter mutation in PIGM causes inherited glycosylphosphatidylinositol deficiency. Nat Med, 12:846-51.

Araten DJ, Bessler M, McKenzie S, et al. 2002. Dynamics of hematopoiesis in paroxysmal nocturnal hemoglobinuria (PNH): no evidence for intrinsic growth advantage of PNH clones. Leukemia, 16:2243-8.
Araten DJ, Nafa K, Pakdeesuwan K, et al. 1999. Clonal populations of hematopoietic cells with paroxysmal nocturnal hemoglobinuria genotype and phenotype are present in normal individuals. Proc Natl Acad Sci USA, 96:5209-14.

Araten DJ, Swirsky D, Karadimitris A, et al. 2001. Cytogenetic and morphological abnormalities in paroxysmal nocturnal haemoglobinuria. Br J Haematol, 115:360-8.

Audebert HJ, Planck J, Eisenburg M, et al. 2005. Cerebral ischemic infarction in paroxysmal nocturnal hemoglobinuria: report of 2 cases and updated review of 7 previously published patients. $J$ Neurol, 252:1379-86.

Bemba M, Guardiola P, Garderet L, et al. 1999. Bone marrow transplantation for paroxysmal nocturnal haemoglobinuria. Br J Haematol, 105:366-8.

Bessler M, Hillmen P, Longo L, et al. 1994. Genomic organization of the $\mathrm{X}$-linked gene (PIG-A) that is mutated in paroxysmal nocturnal haemoglobinuria and of a related autosomal pseudogene mapped to $12 \mathrm{q} 21$. Hum Mol Genet, 3:751-7.

Boccuni P, Del Vecchio L, Di Noto R, et al. 2000. Glycosyl phosphatidylinositol (GPI)-anchored molecules and the pathogenesis of paroxysmal nocturnal hemoglobinuria. Crit Rev Oncol Hematol, 33:25-43.

Bourantas K. 1994. High-dose recombinant erythropoietin and low-dose corticosteroids for treatment of anemia in paroxysmal nocturnal hemoglobinuria. Acta Haematol, 91:62-5.

Brodsky RA, Mukhina GL, Li S, et al. 2000. Improved detection and characterization of paroxysmal nocturnal hemoglobinuria using fluorescent aerolysin. Am J Clin Pathol, 114:459-66.

Brodsky RA, Young NS, Antonioli E, et al. 2008. Multicenter phase III study of the complement inhibitor eculizumab for the treatment of patients with paroxysmal nocturnal hemoglobinuria. Blood, 111:1840-7.

Burge J, Nicholson-Weller A, Austen KF. 1981. Isolation of C4-binding protein from guinea pig plasma and demonstration of its function as a control protein of the classical complement pathway $\mathrm{C} 3$ convertase. J Immunol, 126:232-5.

Cacciapuoti C, Terrazzano G, Barone L, et al. 2007. Glycosyl-phosphatidylinositol-defective granulocytes from paroxysmal nocturnal haemoglobinuria patients show increased bacterial ingestion but reduced respiratory burst induction. Am J Hematol, 82:98-107.

Chen G, Zeng W, Maciejewski JP, et al. 2005. Differential gene expression in hematopoietic progenitors from paroxysmal nocturnal hemoglobinuria patients reveals an apoptosis/immune response in 'normal' phenotype cells. Leukemia, 19:862-8.

Christmas SE, de la Mata Espinosa CT, Halliday D, et al. 2006. Levels of expression of complement regulatory proteins CD46, CD55 and CD59 on resting and activated human peripheral blood leucocytes. Immunology, 119:522-8.

Dufour C, Corcione A, Svahn J, et al. 2001. Interferon gamma and tumour necrosis factor alpha are overexpressed in bone marrow T lymphocytes from paediatric patients with aplastic anaemia. Br J Haematol, 115:1023-31.

Dunn DE, Liu JM, Young NS. 2000. Paroxysmal nocturnal hemoglobinuria. In Young NS (ed). Bone Marrow Failure Syndromes. Philadelphia: WB Saunders Company. p 99-121.

Endo M, Ware RE, Vreeke TM, et al. 1996. Molecular basis of the heterogeneity of expression of glycosyl phosphatidylinositol anchored proteins in paroxysmal nocturnal hemoglobinuria. Blood, 87:2546-57.

Gargiulo L, Lastraioli S, Cerruti G, et al. 2007. Highly homologous T-cell receptor beta sequences support a common target for autoreactive $\mathrm{T}$ cells in most patients with paroxysmal nocturnal hemoglobinuria. Blood, 109:5036-42.

Goebel J, Forrest K, Flynn D, et al. 2002. Lipid rafts, major histocompatibility complex molecules, and immune regulation. Hum Immunol, 63:813-20.

Hall C, Richards S, Hillmen P. 2003. Primary prophylaxis with warfarin prevents thrombosis in paroxysmal nocturnal hemoglobinuria $(\mathrm{PNH})$. Blood, 102:3587-91.

Harrington WJ Sr, Kolodny R, Horstmann LL, et al. 1997. Danazol for paroxysmal nocturnal hemoglobinuria. Am J Hematol, 54:149-54. 
Hauser AC, Brichta A, Pabinger-Fasching I, et al. 2003. Fibrinolytic therapy with rt-PA in a patient with paroxysmal nocturnal hemoglobinuria and Budd-Chiari syndrome. Ann Hematol, 82:299-302.

Hertenstein B, Wagner B, Bunjes D, et al. 1995. Emergence of CD52-, phosphatidylinositolglycan-anchor-deficient $\mathrm{T}$ lymphocytes after in vivo application of Campath-1H for refractory B-cell non-Hodgkin lymphoma. Blood, 86:1487-92.

Hill A, Hillmen P, Richards SJ, et al. 2005a. Sustained response and longterm safety of eculizumab in paroxysmal nocturnal hemoglobinuria. Blood, 106:2559-65.

Hill A, Ridley SH, Esser D, et al. 2006. Protection of erythrocytes from human complement-mediated lysis by membrane-targeted recombinant soluble CD59: a new approach to PNH therapy. Blood, 107:2131-7.

Hill A, Rother RP, Hillmen P. 2005b. Improvement in the symptoms of smooth muscle dystonia during eculizumab therapy in paroxysmal nocturnal hemoglobinuria. Haematologica, 90:ECR40.

Hill A, Richards SJ, Hillmen P. 2007a. Recent developments in the understanding and management of paroxysmal nocturnal haemoglobinuria. Br J Haematol, 137:181-92.

Hill A, Richards SJ, Rother RP, et al. 2007b. Erythopoietin treatment during complement inhibition with eculizumab in a patient with paroxysmal nocturnal hemoglobinuria. Haematologica, 92:ECR14.

Hillmen P, Hall C, Marsh JC, et al. 2004. Effect of eculizumab on hemolysis and transfusion requirements in patients with paroxysmal nocturnal hemoglobinuria. N Engl J Med, 350:552-9.

Hillmen P, Lewis SM, Bessler M, et al. 1995. Natural history of paroxysmal nocturnal hemoglobinuria. $N$ Engl J Med, 333:1253-8.

Hillmen P, Muus P, Duhrsen U, et al. 2007. Effect of the complement inhibitor eculizumab on thromboembolism in patients with paroxysmal nocturnal hemoglobinuria. Blood, 110:4123-8.

Hillmen P, Young NS, Schubert J, et al. 2006. The complement inhibitor eculizumab in paroxysmal nocturnal hemoglobinuria. $N$ Engl $J$ Med, 355:1233-43

Hirose S, Ravi L, Prince GM, et al. 1992. Synthesis of mannosylglucosaminylinositol phospholipids in normal but not paroxysmal nocturnal hemoglobinuria cells. Proc Natl Acad Sci USA, 89:6025-9.

Holguin MH, Wilcox LA, Bernshaw NJ, et al. 1989. Relationship between the membrane inhibitor of reactive lysis and the erythrocyte phenotypes of paroxysmal nocturnal hemoglobinuria. J Clin Invest, 84:1387-94.

Hu R, Mukhina GL, Piantadosi S, et al. 2005. PIG-A mutations in normal hematopoiesis. Blood, 105:3848-54.

Hugel B, Socié G, Vu T, et al. 1999. Elevated levels of circulating procoagulant microparticles in patients with paroxysmal nocturnal hemoglobinuria and aplastic anemia. Blood, 93:3451-6.

Issaragrisil S, Piankijagum A, Tang.Naitrisorana Y. Corticosteroid therapy in paroxysmal nocturnal hemoglobinuria. Am J Hematol, 25:77-83.

Jasinski M, Keller P, Fujiwara Y, et al. 2001. GATA1-Cre mediates Piga gene inactivation in the erythroid/megakaryocytic lineage and leads to circulating red cells with a partial deficiency in glycosyl phosphatidylinositol-linked proteins (paroxysmal nocturnal hemoglobinuria type II cells). Blood, 98:2248-55.

Karadimitris A, Manavalan JS, Thaler HT, et al. 2000. Abnormal T-cell repertoire is consistent with immune process underlying the pathogenesis of paroxysmal nocturnal hemoglobinuria. Blood, 96:2613-20.

Katayama Y, Hiramatsu Y, Kohriyama H. 2001. Monitoring of CD59 expression in paroxysmal nocturnal hemoglobinuria treated with danazol. Am J Hematol, 68:280-3.

Keller P, Payne JL, Tremml G, et al. 2001. FES-Cre targets phosphatidylinositol glycan class A (PIGA) inactivation to hematopoietic stem cells in the bone marrow. J Exp Med, 194:581-9.

Kook H, Risitano AM, Zeng W, et al. 2002 Changes in T-cell receptor VB repertoire in aplastic anemia: effects of different immunosuppressive regimens. Blood, 99:3668-75.

Luzzatto L, Bessler M. 1996. The dual pathogenesis of paroxysmal nocturnal hemoglobinuria. Curr Opin Hematol, 3:101-10.

Luzzatto L, Bessler M, Rotoli B. 1997. Somatic mutations in paroxysmal nocturnal hemoglobinuria: a blessing in disguise? Cell, 88:1-4.
Maciejewski JP, Sloand EM, Sato T, et al. 1997. Impaired hematopoiesis in paroxysmal nocturnal hemoglobinuria/aplastic anemia is not associated with a selective proliferative defect in the glycosylphosphatidylinositolanchored protein-deficient clone. Blood, 89:1173-81.

Mahoney JF, Urakaze M, Hall S, et al. 1992. Defective glycosylphosphatidylinositol anchor synthesis in paroxysmal nocturnal hemoglobinuria granulocytes. Blood, 79:1400-3.

Matis LA and Rollins SA.1995. Complement-specific antibodies: designing novel anti-inflammatories. Nat Med, 1:839-42.

McMullin MF, Hillmen P, Elder GE, et al. 1996. Serum erythropoietin level in paroxysmal nocturnal hemoglobinuria: implications for therapy. Br J Haematol, 92:815-7.

McMullin MF, Hillmen P, Jackson J, et al. 1994. Tissue plasminogen activator for hepatic vein thrombosis in paroxysmal nocturnal haemoglobinuria. J Intern Med, 235:85-9.

Medof ME, Kinoshita T, Nussenzweig V. 1984. Inhibition of complement activation on the surface of cells after incorporation of decay-accelerating factor (DAF) into their membranes. $J$ Exp Med, 160:1558-78.

Miyata T, Takeda J, Iida Y, et al. 1993. The cloning of PIG-A, a component in the early step of GPI-anchor biosynthesis. Science, 259:1318-20.

Moyo VM, Mukhina GL, Garrett ES, et al. 2004. Natural history of paroxysmal nocturnal haemoglobinuria using modern diagnostic assays. Br J Haematol, 126:133-8.

Murakami Y, Kosaka H, Maeda Y, et al. 2002. Inefficient response of $\mathrm{T}$ lymphocytes to glycosylphosphatidylinositol anchor-negative cells: implications for paroxysmal nocturnal hemoglobinuria. Blood, 100:4116-22.

Nafa K, Bessler M, Deeg HJ, et al. 1998. New somatic mutation in the PIG-A gene emerges at relapse of paroxysmal nocturnal hemoglobinuria. Blood, 92:3422-7.

Nafa K, Bessler M, Mason P, et al. 1996. Factor V Leiden mutation investigated by amplification created restriction enzyme site (ACRES) in PNH patients with and without thrombosis. Haematologica, 81:540-2.

Nagakura S, Ishihara S, Dunn DE, et al. 2002. Decreased susceptibility of leukemic cells with PIG-A mutation to natural killer cells in vitro. Blood, 100:1031-7.

Nakao S, Takami A, Takamatsu H, et al. 1997. Isolation of a T-cell clone showing HLA-DRB $1 * 0405$-restricted cytotoxicity for hematopoietic cells in a patient with aplastic anemia. Blood, 89:3691-9.

Nicholson-Weller A, March JP, Rosenfeld SI, et al. 1983. Affected erythrocytes of patients with paroxysmal nocturnal hemoglobinuria are deficient in the complement regulatory protein, decay accelerating factor. Proc Natl Acad Sci USA, 80:5066-70.

Nicholson-Weller A, Spicer DB, Austen KF, et al. 1985. Deficiency of the complement regulatory protein, "decay-accelerating factor," on membranes of granulocytes, monocytes, and platelets in paroxysmal nocturnal hemoglobinuria. $N$ Engl J Med, 312:1091-7.

Ninomiya H, Hasegawa Y, Nagasawa T, et al. 1997. Excess soluble urokinase-type plasminogen activator receptor in the plasma of patients with paroxysmal nocturnal hemoglobinuria inhibits cell-associated fibrinolytic activity. Int $J$ Hematol, 65:285-91.

Nishimura Ji J, Hirota T, Kanakura Y, et al. 2002. Long-term support of hematopoiesis by a single stem cell clone in patients with paroxysmal nocturnal hemoglobinuria. Blood, 99:2748-51.

Nishimura J, Kanakura Y, Ware RE, et al. 2004. Clinical course and flow cytometric analysis of paroxysmal nocturnal hemoglobinuria in the United States and Japan. Medicine (Baltimore), 83:193-207.

Okada N, Harada R, Okada H. 1990. Erythrocytes of patients with paroxysmal nocturnal haemoglobinuria acquire resistance to complement attack by purified $20-\mathrm{kD}$ homologous restriction factor. Clin Exp Immunol, 80:109-13.

Olsen SB, Tang DB, Jackson MR, et al. 1996. Enhancement of platelet deposition by cross-linked hemoglobin in a rat carotid endarterectomy model. Circulation, 93:327-32.

Oni BS, Osunkoya BO, Luzzatto L. 1970. Paroxysmal nocturnal hemoglobinuria: evidence for monoclonal origin of abnormal red cells. Blood, $36: 145-52$. 
Parker C, Omine M, Richards S, et al; International PNH Interest Group. 2005. Diagnosis and management of paroxysmal nocturnal hemoglobinuria. Blood, 106:3699-709.

Peffault de Latour R, Mary JY, Salanoubat C, et al. 2006. Paroxysmal Nocturnal Hemoglobinuria (PNH): Long-Term Epidemiological Study. Blood, 108:291a.

Plasilova M, Risitano A, Maciejewski JP. 2003. Application of the molecular analysis of the T-cell receptor repertoire in the study of immunemediated hematologic diseases. Hematology, 8:173-81.

Plasilova M, Risitano AM, O'Keefe CL, et al. 2004. Shared and individual specificities of immunodominant cytotoxic $\mathrm{T}$ cell clones in Paroxysmal Nocturnal Hemoglobinuria as determined by molecular analysis. Exp Hematol, 32:261-9.

Poggi A, Negrini S, Zocchi MR, et al. 2005. Patients with paroxysmal nocturnal hemoglobinuria have a high frequency of peripheral-blood $\mathrm{T}$ cells expressing activating isoforms of inhibiting superfamily receptors. Blood, 106:2399-408.

Pramoonjago P, Pakdeesuwan K, Siripanyaphinyo U, et al. 1999. Genotypic, immunophenotypic and clinical features of Thai patients with paroxysmal nocturnal haemoglobinuria. Br J Haematol, 105:497-504.

Raiola AM, Van Lint MT, Lamparelli T, et al. 2000. Bone marrow transplantation for paroxysmal nocturnal hemoglobinuria. Haematologica, 85:59-62.

Richards SJ, Rawstron AC, Hillmen P. 2000. Application of flow cytometry to the diagnosis of paroxysmal nocturnal hemoglobinuria. Cytometry, 42:223-33.

Risitano AM, Hill A, Ricci P, et al. 2007. Paroxysmal nocturnal hemoglobinuria $(\mathrm{PNH})$ in the eculizumab era: the bedside and beyond Haematologica, 92(s3):89a.

Risitano AM, Hillmen P, Barbano F, et al. 2006. The complement inhibitor eculizumab to treat paroxysmal nocturnal hemoglobinuria patients: a single center experience within the Triumph, Shepherd and Extension studies. Haematologica, 91(s3):135a.

Risitano AM, Kook H, Zeng W, et al. 2002. Oligoclonal and polyclonal CD4 and CD8 lymphocytes in aplastic anemia and paroxysmal nocturnal hemoglobinuria measured by $\mathrm{V} \beta \mathrm{CDR} 3$ spectratyping and flow cytometry. Blood, 100:178-83.

Risitano AM, Maciejewski JP, Green S, et al. 2004. In vivo dominant immune responses in aplastic anemia patients: molecular tracking of putatively pathogenic T cells by TCR $\beta$-CDR3 sequencing. Lancet, 364:353-63.

Risitano AM, Maciejewski JP, Muranski P, et al. 2005. Large granular lymphocyte (LGL)-like clonal expansions in paroxysmal nocturnal hemoglobinuria (PNH) patients. Leukemia, 19:217-22.

Rosenfeld SI, Jenkins DE Jr, Leddy JP. 1985. Enhanced reactive lysis of paroxysmal nocturnal hemoglobinuria erythrocytes by C5b-9 does not involve increased $\mathrm{C} 7$ binding or cell-bound C3b. J Immunol, 134:506-11.

Rosse WF. 1982. Treatment of paroxysmal nocturnal hemoglobinuria. Blood, 60:20-3.

Rosse WF. 1990. Phosphatidylinositol-linked proteins and paroxysmal nocturnal hemoglobinuria. Blood, 75:1595-601.

Rosse WF. 1996. Epidemiology of PNH. Lancet, 348:573-7.

Rosse WF, Dacie JV. 1966. Immune lysis of normal human and paroxysmal nocturnal hemoglobinuria $(\mathrm{PNH})$ red blood cells. I. The sensitivity of PNH red cells to lysis by complement and specific antibody. J Clin Invest, 45:736-48.

Rosti V, Tremml G, Soares V, et al. 1997. Murine embryonic stem cells without pig-a gene activity are competent for hematopoiesis with the PNH phenotype but not for clonal expansion. $J$ Clin Invest, 100:1028-36.

Rother RP, Bell L, Hillmen P, et al. 2005. The clinical sequelae of intravascular hemolysis and extracellular plasma hemoglobin: a novel mechanism of human disease. JAMA, 293:1653-62.

Rother RP, Rollins SA, Mojcik CF, et al. 2007. Discovery and development of the complement inhibitor eculizumab for the treatment of paroxysmal nocturnal hemoglobinuria. Nat Biotechnol, 25:1256-64.
Rotoli B, Bessler M, Alfinito F, et al. 1993. Membrane proteins in paroxysmal nocturnal haemoglobinuria. Blood Rev, 7:75-86.

Rotoli B, Luzzatto L. 1989. Paroxysmal nocturnal haemoglobinuria. Baillieres Clin Haematol, 2:113-38.

Rotoli B, Robledo R, Luzzatto L. 1982. Decreased number of circulating BFU-Es in paroxysmal nocturnal hemoglobinuria. Blood, 60:157-9.

Rotoli B, Robledo R, Scarpato N, et al. 1984. Two populations of erythroid cell progenitors in paroxysmal nocturnal hemoglobinuria. Blood, 64:847-51.

Rotoli B, Nafa D, Risitano AM. 2006. Paroxysmal nocturnal hemoglobinuria. In: Runge MD and Patterson C, ed. Principles of Molecolar Medicine, 2nd edition. Philadelphia, Humana Press. p 838-47.

Sanchez-Valle E, Morales-Polanco MR, Gomez-Morales E, et al. 1993. Treatment of paroxysmal nocturnal hemoglobinuria with antilymphocyte globulin. Rev Invest Clin, 45:457-61.

Santarone S, Di Bartolomeo E, Bacigalupo, et al. 2008. Long-term results of a GITMO retrospective study on haematopoietic stem cell transplantation for paroxysmal nocturnal hemoglobinura. Bone Marrow Transplant, 41(S1): 19.

Saso R, Marsh J, Cevreska L, et al. 1999. Bone marrow transplants for paroxysmal nocturnal haemoglobinuria. Br J Haematol, 104:392-6.

Sato T, Selleri C, Anderson S, et al. 1997. Expression and modulation of cellular receptors for interferon-gamma, tumour necrosis factor, and Fas on human bone marrow CD34+ cells. Br J Haematol, 97:356-65.

Schafer A, Wiesmann F, Neubauer S, et al. 2004. Rapid regulation of platelet activation in vivo by nitric oxide. Circulation, 109:1819-22.

Selvaraj P, Rosse WF, Silber R, et al. 1988. The major Fc receptor in blood has a phosphatidylinositol anchor and is deficient in paroxysmal nocturnal haemoglobinuria. Nature, 333:565-7.

Shichishima T, Saitoh Y, Terasawa T, et al. 1999. Complement sensitivity of erythrocytes in a patient with inherited complete deficiency of CD59 or with the Inab phenotype. Br J Haematol, 104:303-6.

Shin ML, Hänsch G, Hu VW, et al. 1986. Membrane factors responsible for homologous species restriction of complement-mediated lysis: evidence for a factor other than DAF operating at the stage of C8 and C9. J Immunol, 136:1777-82.

Simak J, Holada K, Risitano AM, et al. 2004. Elevated counts of circulating endothelial membrane microparticles in paroxysmal nocturnal hemoglobinuria indicate inflammatory status and ongoing stimulation of vascular endothelium. Br J Hemat, 125:804-13.

Sloand E, Maciejewski JP, Tisdale J, et al. 2002. Intracellular Interferon- $\gamma$ (IFN- $\gamma$ ) in circulating and marrow $\mathrm{T}$ cells detected by flow cytometry and the response to immunosuppressive therapy in patients with aplastic anemia. Blood, 100:3129-35.

Sloand EM, More K, Shah S, et al. 2006. Soluble urokinase plasminogen activator receptor is increased in patients with paroxysmal nocturnal hemoglobinuria $(\mathrm{PNH})$ and thrombosis and inhibits plasmin generation in vitro and promotes thrombosis in the mouse model. Blood, 108:422a.

Socie G, Mary JY, de Gramont A, et al. 1996. Paroxysmal nocturnal haemoglobinuria: long-term follow-up and prognostic factors. French Society of Haematology. Lancet, 348:573-7.

Stebler C, Tichelli A, Dazzi H, et al. 1990. High-dose recombinant human erythropoietin for treatment of anemia in myelodysplastic syndromes and paroxysmal nocturnal hemoglobinuria: a pilot study. Exp Hematol, 18:1204-8.

Sutherland DR, Kuek N, Davidson J, et al. 2007. Diagnosing PNH with FLAER and multiparameter flow cytometry. Cytometry B Clin Cytom, 72:167-77.

Takahashi M, Takeda J, Hirose S, et al. 1993. Deficient biosynthesis of $\mathrm{N}$-acetylglucosaminyl-phosphatidylinositol, the first intermediate of glycosyl phosphatidylinositol anchor biosynthesis, in cell lines established from patients with paroxysmal nocturnal hemoglobinuria. $J$ Exp Med, 177:517-21.

Takeda J, Miyata T, Kawagoe K, et al. 1992. Deficiency of the GPI anchor caused by a somatic mutation of the PIG-A gene in paroxysmal nocturnal hemoglobinuria. Cell, 73:703-11. 
Tichelli A, Gratwohl A, Nissen C, et al. 1992. Morphology in patients with severe aplastic anemia treated with antilymphocyte globulin. Blood, 80:337-45.

van der Schoot CE, Huizinga TW, van 't Veer-Korthof ET, et al. 1990. Deficiency of glycosyl-phosphatidylinositol-linked membrane glycoproteins of leukocytes in paroxysmal nocturnal hemoglobinuria, description of a new diagnostic cytofluorometric assay. Blood, 76:1853-9.

Wiedmer T, Hall SE, Ortel TL, et al. 1993. Complement-induced vesiculation and exposure of membrane prothrombinase sites in platelets of paroxysmal nocturnal hemoglobinuria. Blood, 82:1192-6.

Wilcox LA, Ezzell JL, Bernshaw NJ, et al. 1991. Molecular basis of the enhanced susceptibility of the erythrocytes of paroxysmal nocturnal hemoglobinuria to hemolysis in acidified serum. Blood, 78:820-9.

Yamashina M, Ueda E, Kinoshita T, et al. 1990. Inherited complete deficiency of 20-kilodalton homologous restriction factor (CD59) as a cause of paroxysmal nocturnal hemoglobinuria. N Engl J Med, 323:1184-9.
Young NS, Maciejewski JP. 2000. Genetic and environmental effects in paroxysmal nocturnal hemoglobinuria: this little PIG-A goes "Why? Why? Why?". J Clin Invest, 106:637-41.

Zalman LS, Wood LM, Müller-Eberhard HJ. 1986. Isolation of a human erythrocyte membrane protein capable of inhibiting expression of homologous complement transmembrane channels. Proc Natl Acad Sci USA, 83:6975-9.

Zalman LS, Wood LM, Frank MM, et al. 1987. Deficiency of the homologous restriction factor in paroxysmal nocturnal hemoglobinuria. J Exp Med, 165:572-7.

Zeng W, Maciejewski JP, Chen G, et al. 2001. Limited heterogeneity of $\mathrm{T}$-cell receptor VB usage in aplastic anemia. J Clin Invest, 108:765-73.

Zoumbos N, Gascon P, Trost S, et al. 1985. Circulating activated suppressor T lymphocytes in aplastic anemia. N Engl J Med, 312:257-65. 